\title{
Monitoring paint and primer samples using multispectral and hyperspectral imaging techniques
}

\author{
Nawarat Kaew-On ${ }^{\mathrm{a}}$, Pichayada Katemake ${ }^{\mathrm{a}, *}$, Alain Trémeau ${ }^{\mathrm{b}}$ \\ a Department of Printing and Imaging Technology, Faculty of Science, Chulalongkorn University, \\ Patumwan, Bangkok 10330 Thailand \\ b Univ Lyon, UJM Saint-Étienne, CNRS, Laboratoire Hubert Curien UMR 5516 France \\ *Corresponding author, e-mail: pichayada.k@chula.ac.th
}

Received 22 Oct 2019

Accepted 28 Mar 2020

\begin{abstract}
Multispectral imaging (MSI) and hyperspectral imaging (HSI) are non-invasive techniques that have been used to map and identify pigments, binders and retouches on work of arts. The spectral reflectance obtained from these techniques could be used for matching pigments. In this research we aimed to use these 2 techniques for monitoring the change of paint samples after they were systematically exposed by heat, moisture and light. Three primer samples, developed in our previous research, were applied on the acid and lignin free substrate, wet film thickness of $40 \mu \mathrm{m}$, and left to dry for 30 days in a controlled condition. Five selected acrylic paints and 4 pastels, developed in accordance with Thai color names' dictionary, were similarly applied on the same type of substrate. All samples were exposed to moist heat at $50^{\circ} \mathrm{C}$ and $60 \%$ RH up to 40 days. The rest of the paint samples were exposed to full spectrum of sunlight for $1,10,100$, and $1000 \mathrm{~h}$. The spectral reflectances of samples were recorded. In monitoring sample using MSI, the samples were captured with 10 bandwidths filters, 400-700 nm. The HSI, 400-1000 nm, with false color post process to images was employed to monitor the change of paint samples after exposing to UV of $1-50 \mathrm{~h}$. The RGB and CIEXYZ data of MSI images were extracted and analysed. The colorimetric data could be used to examine the color change of paint samples. The lightness difference obtained from the MSI images showed distinct result in primers.
\end{abstract}

KEYWORDS: multispectral imaging, hyperspectral imaging, colorimetric values

\section{INTRODUCTION}

Colors used in Thai mural and arts have their own style and name. The paint media traditionally was tempera made of natural pigment ground to fine powder and mixed with gum made of plants or animals particularly gum Arabic. In Thailand, degradation of mural paintings has been proceeding because of both environment parameters and improper restoration. Some factors that lead to mistakes in restoration are lack of registration of paintings and recoding of the traditional Thai color formulations and names. Elder artists passed their knowledge to younger generation in the artists' painting studio which might be different among them. There is no general accepted recipe among artists to produce any of these colors by means of pigment proportions and dilutions $[1,2]$. Only a few artists today possess the knowledge inherited from their elders to produce traditional Thai colors from natural pigments $[1,2]$. The report by the Crown Property Bureau also mentioned that traditional recipes of the paints and primers have disap- peared [3]. The report did not find any scientific or significant statistical data. Therefore, obtaining the correct Thai color cannot be guaranteed for restoration and re-creation.

Recently, commercial pigments and acacia gum are simply mixed for painting the murals. Sometimes the famous Rembrandt pastels are selected to obtain specific shade. Moreover, imported acrylic paints have been used for most of Thai murals for some times.

In our preliminary research [4], we focused on making acrylic paints and pastels having the same color as specified by Katemake and Preda [1]. Acrylic paints were made of synthetic pigments and styrene/acrylic resin as binder, while the pastels were made of powder pigments and gum tragacanth as binder. These types of paints were aimed for re-creation of Thai mural paintings and some other artifacts such as Khon masks. Practically they could be used satisfactorily by artists after testing.

In general, almost all of studies that identified paint deterioration employed multi-methods 
to analyse results. Advance microscopy (scanning electron microscopy (SEM)/energy dispersive X-Ray (EDX), atomic force microscopy (AFM) and spectroscopy (UV-VIS and FTIR) techniques were frequently utilised $[5,6]$. These techniques are invasive except for the portable EDXRF.

In this report we were interested in using MSI and HSI devices to record and analyse changes of materials before and after ageing. It is well known that MSI is a non-invasive technique that has been employed to map and identify pigments, binder, retouches on work of arts and also used to visually improve old and faded documents in painting and arts' conservation field [7]. It is an image data captured within specific wavelength ranges across the electromagnetic spectrum. The wavelengths can be separated by filters or by the use of instruments that are sensitive to particular wavelengths, including light from frequencies beyond the visible light such as infrared (IR) and ultraviolet (UV). Sampling important work of art is normally not permitted, only non-invasive technique is allowed to conduct and MSI can serve this requirement. In addition, this method is simple and affordable. The devices are lightweight and small. Furthermore, it gives a low-cost survey even for large area [7, 8]. Using MSI for art conservation is simply performed. It can be equipped with a variety of equipment setup, basically a monochromatic camera and a set of band-pass filters. There are many types of MSI; MSI in this concept is different from MSI technique that Cosentino [7] did for identifying pigments.

HSI is similar to MSI but the number of captured images is much more than the MSI. HSI technique can capture every nanometer, wavelength range from visible to mid infrared. Consequently, it provides a great number of information than MSI.

Though acrylic base paint media has been used for only 60-70 years [9-12] but there are numerous literatures presented degradation of art works using this synthetic material. Mecklenburg presented that at the low temperature acrylic paint film was stable without any damage due to their increasing of bulk material strength. At the temperature below $\mathrm{Tg}$, acrylic paint film became brittle and lost significant ability to elongate [13]. In addition, relative humidity (\%RH) also increased free-swelling-strain. In Mecklenburg's study, it was also mentioned that the change of RH range (10-80\%) caused a larger mechanical property change than the change of temperature range $\left(-28^{\circ} \mathrm{C}\right.$ to $\left.27^{\circ} \mathrm{C}\right)$. Ziraldo et al [14] studied the influence of temperature and humidity on swelling and surfactant migration in acrylic emulsion paint film. The results showed that when the temperature rose above $20^{\circ} \mathrm{C}$ or as $\% \mathrm{RH}$ increased above $40 \%$, the surfactant moved from the surface into the paint film. This made the paint film change in volume and thickness. Above $40 \% \mathrm{RH}$, the films softened and swelled in volume and surfactant moved into the paint film. Below $40 \% \mathrm{RH}$, surfactant moved out from the paint body to the paint surface. This agreed with the Smithsonian Institution's $\mathrm{RH}$ and temperature guideline that are $45 \pm 8 \% \mathrm{RH}$ and $21 \pm 2{ }^{\circ} \mathrm{C}$, respectively [14].

The stability to outdoor weathering condition of vinyl, acrylic and styrene acrylic formulations used in waterborne paint in contemporary mural were also studied by means of photochemical degradation. Di Crescenzo et al [11] reported that stability of acrylic and styrene-acrylic waterborne paints used to create exterior mural was more stable than the vinyl waterborne paints. It showed that the degradation of binders led to variations in the morphology of the paint layers, which led to color changes. Stability to photo-oxidative of a series of red acrylic paints was studied after accelerated UV light exposure was applied for a total time of $1200 \mathrm{~h}$. The results of FT-IR spectroscopy showed structure changes of cadmium, quinacridone scarlet, primary magenta and vermilion. Colorimetric measurements indicated the reduction in the absorption of visible light due to photo-degradation of the pigment structure. We could see that the investigation of the degradation and deterioration used the invasive technique or the combination of invasive and non-invasive techniques.

In this research, we investigated if the noninvasive techniques alone could be used for monitoring the deterioration of paints and described the limitations of the techniques. The colorimetric data before and after being deteriorated were analysed.

\section{MATERIALS AND METHODS}

\section{Preparation of samples}

We selected 3 types of sample from our previous research and re-formulated them with the same proportions [15]. Firstly, 3 primers were: (1) The traditional primer ( $\mathrm{T})$, made of tamarind glue as binder mixed with white clay as pigment; (2) the 1st modern primer (NTA-Ag), made of styrene/acrylic binder, titanium dioxide as white pigment and aluminium silicate as filler and (3) the 2nd modern primer (NA-Ag), made of styrene/acrylic binder and aluminium silicate as white pigment. The two modern primers contained silver nanoparticle as an 
additive for antibacterial purpose. Information of primer materials was as follows: styrene/acrylic emulsion (PRIMAL, Dow Chemical Company), silver nanoparticle (Prime Nano Co., Ltd, Thailand). titanium dioxide (Duponts Grade R902), and aluminium silicate (Satintone 5HB, BASF Corporation). Secondly, 2 primary colors and 2 Thai style colors of acrylic paint were formulated. They were made of synthetic pigments mixed with styrene/acrylic binder, small percentage of aluminium silicate as filler and other additives needed. The pigments used in 2 primary colors included Colanyl Green GG 131 (phthalocyanine green, PG7) and Colanyl Pink E (quinacridone, PR122). The Thai style colors included "Lueang Rong" and "Khiao Khram" (indigo green). "Lueang Rong" contained Colanyl Green (PG7), Colanyl Oxide Yellow R-TH (PY42), Flexonyl Yellow WF013 (PY13). "Khiao Khram" contained Colanyl Green (PG7), Colanyl PinkE (PR122), and Colanyl Yellow H3G 500 (PY154). Lastly, 2 primary colors and 2 Thai style colors of pastel were formulated. They were made of synthetic pigments mixed with gum tragacanth (Texture CX55 from Ingredient Flavor Co., Ltd Thailand) as binder and calcium carbonate $\left(\mathrm{CaCO}_{3}\right.$, commercial grade) as filler. The pigments of pastel primary colors included Hostoperm Red D3G70 (PR254) and Hostoperm Violet RLS-EF (PV23). The Thai style pastel colors selected were "Dang Sen" (Orange Red) and "Luk Wa". "Dang Sen" contained Hostoperm Orange GR (PO43), Hostoperm Red D3G70 (PR254) and Hostoperm Violet RLS-EF (PV23). "Luk Wa" contained Hostoperm Orange GR (PO43), Hostoperm Pink E M25 (PR122) and Hostoperm Violet RLS-EF (PV23).

\section{Samples for monitoring the change affected by moist and heat}

The primers and acrylic paints were applied directly on canvas substrate with the wet film thickness of $40 \mu \mathrm{m}$. The pastel samples (powder) were mixed with the gum arabic before applying with the same method.

\section{Samples for monitoring the change affected by ultraviolet radiation}

The primers, acrylic paints and the pastel mixtures were applied, with the same method explained previously, on "Fabriano" substrates, $300 \mathrm{~g} / \mathrm{m}^{2}$, a cellulose and cotton paper, acids and lignin free. This paper is not treated with optical brightening agent.

\section{Acceleration of ageing}

After one month of drying in parafilm sealed petridish at room temperature, two different ageing procedures were performed.

(i) Influence of moisture and heat was performed in a climatic chamber at $50{ }^{\circ} \mathrm{C}(\mathrm{T})$ and $60 \% \mathrm{RH}$ for 10, 20, 30 and 40 days. The guidelines for environmental control in cultural institutions written in Consortium for Heritage Collections and their Environment suggested that for hot humid, hot dry and temperate climates, the level of $\mathrm{T}$ and $\mathrm{RH}$ should be kept within the boundaries of $22-28^{\circ} \mathrm{C}$ and $55-70 \%, 22-28^{\circ} \mathrm{C}$ and $40-60 \%$ as well as $18-24^{\circ} \mathrm{C}$ and $45-65 \%$, respectively [16]. T and $\mathrm{RH}$ are interrelated, in the closed space, an increase in $\mathrm{T}$ will produce a decrease in RH. In our study, we accelerated the deterioration by increasing temperature to $50^{\circ} \mathrm{C}$ at the maximum where the $\mathrm{RH}$ could be set at $60 \%$.

(ii) Influence of UV radiation, in this case the samples were put in the Q-Sun Xenon Test Chamber Xe-1 with Xenon Arc Lamp. Test conditions were $0.80 \mathrm{~W} / \mathrm{m}^{2}$ in the visible range at $420 \mathrm{~nm}$ for $1,10,100$ and $1000 \mathrm{~h}$.

\section{Colorimetric measurements}

The spectral reflectances of samples were measured using the Minolta spectroradiometer (CS-1000) under daylight 6500 Kelvin, in the visible range of $380-780 \mathrm{~nm}$ relative to the white standard BYKGardner $\mathrm{GmbH}$. This device did not contact to the sample during the measurement.

\section{Multispectral imaging}

We employed 2 sets of MSI devices. The Sony XCDX71 and the Thorcam cameras were used for UV radiation ageing samples and for moist and heat ageing samples. The filters chosen were from LotOriel France, 31 bandwidth of $10 \mathrm{~nm}$ covered from 400-700 nm. The lamp was D65 ILLUM 4000, Eurosep Instrument 4 and the calibration white patch was PRC Krochmann GmbH. GT20000 Epson scanner and Trichromatic Color Analyzer (TCA) software were used for transforming the RGB of the multispectral images into CIELAB [1].

\section{Hyperspectral imaging}

The type of MSI we used was similar to the type of HSI which we could have stack of images in finer wavelength, $1 \mathrm{~nm}$ interval. We, then, decided to use HSI technique with post processing, such 
(a)

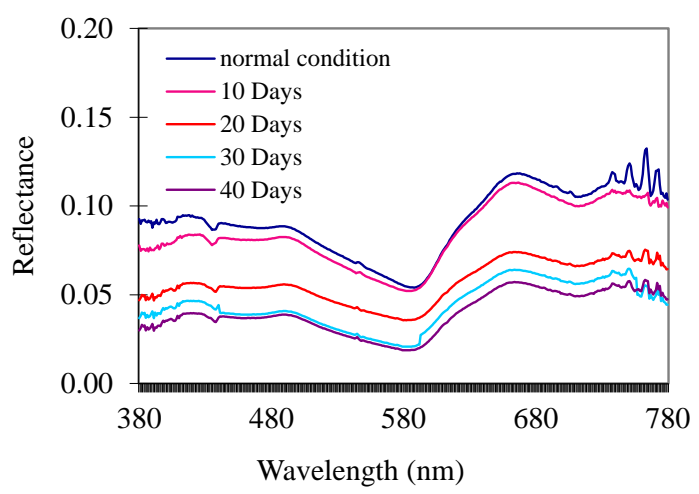

(b)

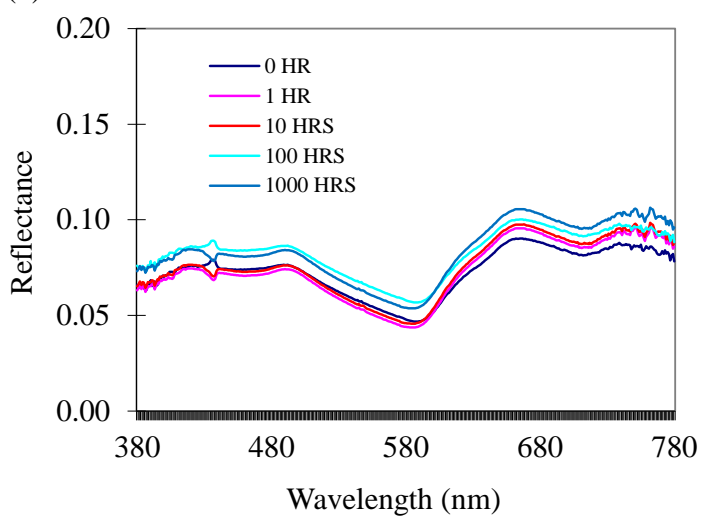

Fig. 1 Spectral reflectance of samples-Colanyl green 131 (PG7), phthalocyanine green, (a) before and after ageing with moist and heat for 10, 20, 30 and 40 days and (b) after ageing with UV radiation for 1, 10, 100 and $1000 \mathrm{~h}$.

as false color, to the HSI images. Additionally, the excitation light included infrared. We used the Video Spectral Comparator, VSC 8000. In HSI mode, flood lighting and visible light plus infrared, 400-1000 $\mathrm{nm}$ was exposed to the samples. To examine infra-red fluorescence using the VSC 8000 , the SPOT (Fluorescence) light source was used. This light source was a high intensity light source that was filtered to provide the user with a choice of excitation wavebands of light.

\section{RESULTS}

From the spectral reflectances measured before and after treatment with moist heat, we calculated the color differences (CIE $\Delta \mathrm{E}_{\mathrm{ab}}^{*}$ ), D65/2 of the samples before and after ageing for 40 days (Table 1 ). The total color differences of 2 primers, NTA-Ag and T are greater than 2, which we could perceive a small color difference. The NTA-Ag looked less yellow than the original sample and the primer $\mathrm{T}$ looked lighter. For the acrylic paints and pastels, the moist heat caused more lightness change than chroma and hue $\left(\Delta \mathrm{a}^{*}\right.$ and $\Delta \mathrm{b}^{*}$ did not change much compared to $\left.\Delta \mathrm{L}^{*}\right)$. On the other hand, ageing by UV radiation caused more change in chroma and hue than lightness (Table 2). The most change in color was found in "Lueang Rong" containing Flexonyl Yellow WF 013 (PY13) having poor lightfastness [17].

The spectral reflectance relates to the colorimetric data (Tables 1 and 2). We might monitor the change through the colorimetric data but the spectral reflectance should be used to confirm. For instance, the spectral reflectance of the Colanyl green 131 (PG7) phthalocyanine green samples, before and after ageing, measured using Konica Minolta CS-1000 spectroradiometer, was shown in Fig. 1.

After ageing with moist heat, the reflectance factors of samples decreased in all visible wavelengths. Ageing with UV radiation ( $0-1000 \mathrm{~h})$ resulted in very small increase of reflectance factors compared to the effect of moist heat. Both ageing, for the duration carried out in this research, did not show a great change in samples with only phthalocyanine green). This could be seen easily using the colorimetric data, calculated from the reflectance factor; it helps in describing color in 3 dimensions: hue, chroma and lightness.

The images obtained from the MSI technique used in this research, visible light from 400-700 nm with filters of 31 bandwidths, were quite difficult to observe the change due to the ageing by moist heat and UV radiation. After the RGB data of images, captured under individual bandwidth, were extracted and converted into CIELAB values and compared, the perceptible lightness difference (CIE $\Delta \mathrm{L}^{*}$ ) could be detected in some wavelengths. The results of all primary colors and some of the mixed pigment paints were shown here because the paints with mixed pigments were dependent on the main pigment of the mixture. The results could be described as follows

1. Primary color, Colanyl Pink E (quinacridone PR122) showed the most decreasing of CIE L* value $\left(\Delta \mathrm{L}^{*}\right)$ of 7.8 , after ageing with moist heat for 40 days, at $540 \mathrm{~nm}$. After ageing with UV radiation for $1000 \mathrm{~h}$ the CIE L* clearly increased more than 10 units at the wavelengths of 560 and $640 \mathrm{~nm}$.

2. Primary color, Colanyl Green GG 131-TH (PG7, 
Table 1 Color differences of samples before and after ageing with moist and heat for 40 days.

\begin{tabular}{|c|c|c|c|c|}
\hline Color sample & $\Delta \mathrm{L}^{*}$ & $\Delta \mathrm{a}^{*}$ & $\Delta \mathrm{b}^{*}$ & $\Delta \mathrm{E}^{*}$ \\
\hline $\begin{array}{l}\text { Acrylic paint } \\
\text { 1. Primary color-Colanyl Pink E (PR122 Quinacridone) } \\
\text { 2. Primary color-Colanyl Green 131 (PG7 Phthalocyanine green) } \\
\text { 3. Khiao Khram-PG7+PR122+Colanyl Yellow H3G 500 (PY154) } \\
\text { 4. Lueang Rong-PG7+Colanyl Oxide Yellow R-TH (PY42)+Flexonyl Yellow WF013 (PY13) }\end{array}$ & $\begin{array}{r}0.56 \\
-1.31 \\
2.07 \\
1.32\end{array}$ & $\begin{array}{r}-0.28 \\
0.55 \\
-0.67 \\
-0.61\end{array}$ & $\begin{array}{r}0.06 \\
0.06 \\
0.15 \\
-0.58\end{array}$ & $\begin{array}{l}0.63 \\
1.42 \\
2.18 \\
1.57\end{array}$ \\
\hline $\begin{array}{l}\text { Pastel } \\
\text { 1. Primary color-Red (PR254 Pyrroles red), } \\
\text { 2. Primary color-Violet (PV23 Dioxazine purple) } \\
\text { 3. Dang Sen-Hostoperm Orange GR (PO43)+PR254+PV23 } \\
\text { 4. Luk Wa-PO43+Hostoperm Pink E M25 (PR122)+PV23 }\end{array}$ & $\begin{array}{l}0.35 \\
1.07 \\
0.14 \\
0.92\end{array}$ & $\begin{array}{r}0.27 \\
0.35 \\
-0.13 \\
-0.09\end{array}$ & $\begin{array}{r}0.27 \\
-0.89 \\
-0.05 \\
0.15 \\
\end{array}$ & $\begin{array}{l}0.52 \\
2.06 \\
0.20 \\
0.94\end{array}$ \\
\hline $\begin{array}{l}\text { Primer } \\
\text { NTA-Ag } \\
\text { NA-Ag } \\
\text { T }\end{array}$ & $\begin{array}{l}0.98 \\
0.37 \\
2.37\end{array}$ & $\begin{array}{r}-0.95 \\
0.12 \\
-1.70\end{array}$ & $\begin{array}{l}-2.18 \\
-1.66 \\
-0.55\end{array}$ & $\begin{array}{l}2.57 \\
1.71 \\
2.97\end{array}$ \\
\hline
\end{tabular}

Table 2 Color differences of samples before and after ageing with UV radiation for $1000 \mathrm{~h}$.

\begin{tabular}{|c|c|c|c|c|}
\hline Color sample & $\Delta \mathrm{L}^{*}$ & $\Delta \mathrm{a}^{*}$ & $\Delta \mathrm{b}^{*}$ & $\Delta \mathrm{E}^{*}$ \\
\hline $\begin{array}{l}\text { Acrylic paint } \\
\text { 1. Primary color-Colanyl Pink E (PR122 Quinacridone) } \\
\text { 2. Primary color-Colanyl green } 131 \text { (PG7 Phthalocyanine green) } \\
\text { 3. Khiao Khram-PG7+PR122+Colanyl Yellow H3G 500 (PY154) } \\
\text { 4. Lueang Rong-PG7+Colanyl Oxide Yellow R-TH (PY42)+Flexonyl Yellow WF } 013 \text { (PY13) }\end{array}$ & $\begin{array}{l}1.03 \\
0.46 \\
2.85 \\
0.80\end{array}$ & $\begin{array}{r}1.76 \\
-1.01 \\
-0.33 \\
1.57\end{array}$ & $\begin{array}{r}-2.78 \\
-0.52 \\
1.14 \\
-20.82\end{array}$ & $\begin{array}{r}3.45 \\
1.23 \\
3.08 \\
20.90\end{array}$ \\
\hline $\begin{array}{l}\text { Pastel } \\
\text { 1. Primary color-Red (PR254 Pyrroles red), } \\
\text { 2. Primary color-Violet (PV23 Dioxazine purple) } \\
\text { 3. Dang Sen-Hostoperm Orange GR (PO43)+PR254+PV23 } \\
\text { 4. Luk Wa-PO43+Hostoperm Pink E M25 (PR122)+PV23 }\end{array}$ & $\begin{array}{r}-1.19 \\
1.35 \\
-0.83 \\
3.18\end{array}$ & $\begin{array}{r}-1.89 \\
0.47 \\
1.34 \\
-1.17 \\
\end{array}$ & $\begin{array}{r}-1.48 \\
-1.43 \\
1.65 \\
0.87 \\
\end{array}$ & $\begin{array}{l}2.68 \\
2.02 \\
2.28 \\
3.50 \\
\end{array}$ \\
\hline $\begin{array}{l}\text { Primer } \\
\text { NTA-Ag } \\
\text { NA-Ag } \\
\text { T }\end{array}$ & $\begin{array}{l}0.87 \\
0.26 \\
0.15\end{array}$ & $\begin{array}{l}-0.10 \\
-0.01 \\
-1.57\end{array}$ & $\begin{array}{l}-2.39 \\
-1.89 \\
-1.62\end{array}$ & $\begin{array}{l}2.54 \\
1.91 \\
2.26\end{array}$ \\
\hline
\end{tabular}

phthalocynanine green) showed decreasing of CIE L* greater than 10 units, after ageing with moist heat for 40 days, at wavelenghts 400 to $580 \mathrm{~nm}$, and increasing at 620 to $640 \mathrm{~nm}$ after exposing to UV for $1000 \mathrm{~h}$.

3. Khiao Khram-PG7+PR122+Colanyl Yellow H3G 500 (PY154) showed increasing of CIE L* greater than 10 units, after ageing with moist heat for 40 days, at $460 \mathrm{~nm}$. After exposing to $\mathrm{UV}$ radiation for $1000 \mathrm{~h}$, the CIE $\mathrm{L}^{*}$ increased the most (8.1) at the $640 \mathrm{~nm}$.

4. Primary color, Hostaperm Red D3G70 (Pyrrole Red PR254) showed the most decreasing of CIE $L^{*}$ value $\left(\Delta L^{*}\right)$ of 8.6 , after ageing with moist heat for 40 days, at $520 \mathrm{~nm}$. After ageing with UV radiation for $1000 \mathrm{~h}$, the CIE L* decreased more than 10 units at $600 \mathrm{~nm}$.

5. Primary color, Violet RLS-EF (Dioxazine purple) did not show great difference in lightness after ageing with moist heat for 40 days. The most $-\Delta \mathrm{L}^{*}$ of 3.81 was found at $440 \mathrm{~nm}$. After ageing with UV radiation for $1000 \mathrm{~h}$, the CIE $\mathrm{L}^{*}$ decreased more than 10 units at 540 to $580 \mathrm{~nm}$ with $10 \mathrm{~nm}$ interval.
6. Dang Sen-Hostoperm Orange GR PO43+PR254 +PV23 did not show great difference in lightness after ageing with moist heat for 40 days. The most $-\Delta \mathrm{L}^{*}$ of 6.70 was found at $500 \mathrm{~nm}$. The $\Delta \mathrm{L}^{*}$ after ageing with UV radiation for $1000 \mathrm{~h}$ was much smaller in all wavelengths.

7. Novel primer NTA-Ag showed increasing in CIE L* of 20.87 at $560 \mathrm{~nm}$ after ageing with moist heat for 40 days. After ageing with UV radiation for $1000 \mathrm{~h}$, the $C I E L^{*}$ decreased more than 10 units at $560 \mathrm{~nm}$.

8. Novel Primer NA-Ag showed increasing in CIE $\mathrm{L}^{*}$ of 14.6 at $560 \mathrm{~nm}$ after ageing with moist heat for 40 days. After ageing with UV radiation for $1000 \mathrm{~h}$, the CIE $\mathrm{L}^{*}$ increased more than 10 units (23.5) at $470 \mathrm{~nm}$.

9. Traditional primer showed increasing in CIE $\mathrm{L}^{*}$ of 11.45 at $540 \mathrm{~nm}$ after ageing with moist heat for 40 days. After ageing with UV radiation for $1000 \mathrm{~h}$, the CIE L* decreased (7.3) the most at $560 \mathrm{~nm}$.

We prepared another set of acrylic paint samples to be used for HSI technique and we did not control the thickness of the paint film as we did using K-bar 

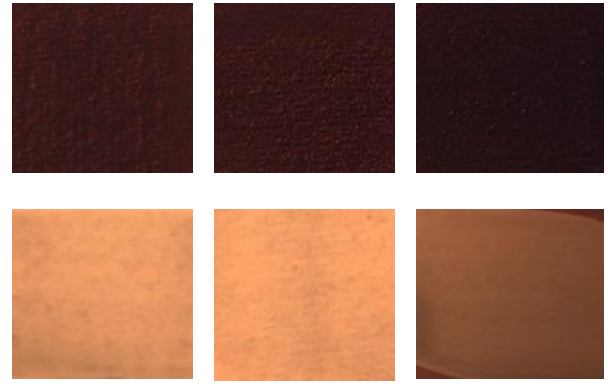

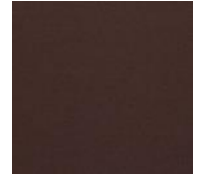

$1 \mathrm{~h}$

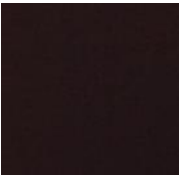

$10 \mathrm{~h}$

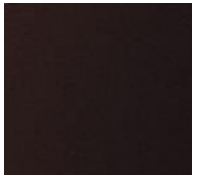

$50 \mathrm{~h}$
Fig. 2 Images of samples, treated with UV radiation for 1,10 and $50 \mathrm{~h}$, captured using IR fluorescence technique; Colanyl Green GG 131 TH (top); Colanyl Pink E-WD500 (middle) and Colanyl Green GG 131 TH + Colanyl Pink E-WD500 (bottom).

as in MSI. One stroke of medium paint brush was painted on the same substrate as we used for MSI. The samples were ageing by the same UV radiation with the duration of 1,10 and $50 \mathrm{~h}$. The captured images, which were similar to MSI, were applied false color image processing. It was found that the differences among HSI false color images of 0,1 , 10 and $50 \mathrm{~h}$ were not perceptible. This might be due to the short duration of UV exposing and the limitation of the VSC 8000 having the visible/NIR $(400-1000 \mathrm{~nm})$. The NIR of about $900-1700 \mathrm{~nm}$ would be more appropriate.

\section{DISCUSSION}

Monitoring the deterioration of important piece of art, using MSI, could be done by qualitative band to band comparison relative to time and by quantitative method. We demonstrated that the colorimetric results obtained from MSI images could be used to approximately assess the cause of change, moist and heat or UV radiation, to particular pigment. Practically, various pigments are used in paintings. They have different chemical structures and properties which are sensitive to moist, heat and light differently, therefore, the analysis must be carried out carefully. Martinez et al [18] developed procedure for comparing between colorimetric image of the same paintings recorded at different times. They monitored the color change of 7 paintings in long-term study. Their procedure could elim- inate possible errors due to imaging set up and imaging equipment when time difference was about 10 years. They claimed that the color difference $\left(\Delta \mathrm{E}^{*} \mathrm{ab}\right)$ between "before" and "after" exact area was less than 4 units and there was no color change. Our colorimetric results shown in Tables 1 and 2 showed $\Delta \mathrm{E}^{*}$ ab less than 3 except for "Lueang Rong" that contained poor lightfastness pigment. We could perceive color difference between "before" and "after" paint sample $(5 \times 5 \mathrm{~cm})$, made with single pigment when the $\Delta \mathrm{E}^{*}$ ab was greater than 2 . This is possible because we compared a pair of single color. The color tolerance of a pair of complex image is greater than that of single color [19].

One of the simulated ageing we used was moist $(60 \% \mathrm{RH})$ and heat $\left(50^{\circ} \mathrm{C}\right)$ with the maximum of 40 days. In terms of colorimetric measurement, high color difference ( $\Delta \mathrm{E}^{*}{ }_{\mathrm{ab}}$ between 2 and 3 ) was found in 2 primers. As mentioned before, this small change might not be perceptible if it is in the real painting but our samples were single color. The MSI results, after converting the RGB of the entire sample to CIELAB, showed that $\Delta \mathrm{L}^{*}$ of "before" and "after" ageing was greater than 10 units. Another more advanced method, claimed by Liang [20], used the PRISMS SWIR hyperspectral imager to monitor moisture in the walls using $1.4 \mu \mathrm{m}$ band. Liang mentioned that the water absorption bands of around 1.4 and $1.9 \mu \mathrm{m}$ could be used to monitor moisture content. We thought that our method could be possible in some types of material. The $\Delta \mathrm{L}^{*}$ obtained from the MSI images of color paint samples were not high compared to the primers'. The lightness difference is less sensitive to human vision system. The lightness tolerance is greater than chroma and hue.

Regarding short exposing time to UV radiation of the samples leading to imperceptible change of the HSI false color images, we thought another technique might help to enhance visualisation. We believe that pigments would fluoresce if they have been stimulated with a light of the requisite wavelength and intensity. We then selected and applied the infra-red fluorescence technique to 3 exposed samples: (1) acrylic paint with Colanyl Green GG 131 pigment, (2) acrylic paint with Colanyl Pink EWD500 and (3) acrylic paint with the mix of (1) and (2). For this technique, we used excitation light from 485-610 nm plus IR. The samples absorbed and reflected some of visible light and emitted IR fluorescent. The reflected visible light was blocked by automatically selected filter, only the emitted IR fluorescent reached the camera sensor. This 
process was carried out by using the Video Spectral Comparator, VSC 8000, the same device used for HSI. It was found that even short period of UV exposing, the infra-red fluorescence could enhance the visualization of the captured images as shown in Fig. 2.

The IR fluorescence is another technique that could be used to monitor the change of arts affected by UV radiation in short period.

\section{CONCLUSION}

The spectroradiometer could be used for monitoring the change of the spectral reflectances of the white primers after moist and heat deterioration. The colorimetric data calculated from the reflectance could be used for detecting the changes in hue, chroma and lightness of acrylic paints and pastels. For the MSI technique, the lightness (CIE L*) of the images obtained from some wavelengths showed perceptible differences before and after moist and heat deterioration. The HSI technique used in the study had a limitation in visible/NIR (400-1000 nm) and the difference of the false color images could not be detected after UV deterioration at the maximum of $50 \mathrm{~h}$. Applying IR fluorescence technique to the same samples, the differences of images before and after UV deterioration could be detected. For the further study, the duration of UV, moist and heat deterioration should be extended.

Acknowledgements: The authors gratefully acknowledge the Thai Research Fund, the Ministry of Science and Technology and Kenton Intertrade Co., Ltd, Thailand (Grant No. PHD57I0074) for financial support.

\section{REFERENCES}

1. Katemake P, Preda RI (2014) Complete study of traditional thai colors used in mural paintings: Traditional Thai color name dictionary. Color Res Appl 39, 616-629.

2. Katemake P, Preda RI, Hoontrakul D (2013) Identification of traditional Thai colours used for mural paintings and Khon masks. Color Res Appl 38, 229-234.

3. Bureau TCP (2013) Rattana Hang Jittragum. In: The Mural Painting in the Pra Buddharattanasatara, the Art in the King Rama 9 Period, p 102. [in Thai]

4. Kaew-on N (2018) Formulations of primer, acrylic paint and pastel in traditional Thai style colour. $\mathrm{PhD}$ thesis, Chulalongkorn Univ, Bangkok, Thailand.

5. Doménech-CarbóMT, Silva MF, Aura-Castro E, Fuster-López L, Kröner S, Martínez-Bazán ML, Más-Barberá X, Mecklenburg MF, et al (2011) Study of behaviour on simulated daylight ageing of artists' acrylic and poly (vinyl acetate) paint films. Anal Bioanal Chem 399, 2921-2937.

6. Papliaka ZE, Andrikopoulos KS, Varella EA (2010) Study of the stability of a series of synthetic colorants applied with styrene-acrylic copolymer, widely used in contemporary paintings, concerning the effects of accelerated ageing. $J$ Cult Herit 11, 381-391.

7. Cosentino A (2015) Multispectral imaging and the art expert. Spectroscopy Eur 27, 6-9.

8. Cosentino A (2014) Identification of pigments by multispectral imaging; a flowchart method. Herit Sci 2, ID 8.

9. Jones FN, Mao W, Ziemer PD, Wiao F, Hayes J, Golden M (2005) Artist paints-an overview and preliminary studies of durability. Prog Org Coat 52, 9-20.

10. Jablonski E, Learner T, Hayes J, Golden M (2004) The conservation of acrylic emulsion paintings: A literature review. Tate Paper, available at: www.tate. org.uk.

11. Di Crescenzo MM, Zendri E, Sánchez-Pons M, FusterLopez L, Yusa-Marco DJ (2014) The use of waterborne paints in contemporary murals: comparing the stability of vinyl, acrylic and styrene-acrylic formulations to outdoor weathering conditions. Polym Degrad Stab 107, 285-293.

12. Learner $\mathrm{T}$ (2000) A review of synthetic binding media in twentieth-century paints. Conserv 24, 96-103.

13. Mecklenburg MF (2007) Determining the Acceptable Ranges of Relative Humidity and Temperature in $\mathrm{Mu}$ seums and Galleries, Part 1, Structural Response to Relative Humidity, Smithsonian Libraries.

14. Ziraldo I, Watts K, Luk A, Lagalane AF, Wolbers RC (2016) The influence of temperature and humidity on swelling and surfactant migration in acrylic emulsion paint films. Stud Conserv 61, 209-221.

15. Kaew-on N, Katemake P, Prasongsuk S (2020) Primer formulations with antibacterial properties for murals. Prog Org Coat 138, ID 105395.

16. Heritage Collections Council (2002) Guidelines for Environmental Control in Cultural Institutions: Consortium for Heritage Collections and Their Environment, Canberra, A.C.T., Australia.

17. Hunger K, Schmidt MU (2019) Industrial Organic Pigments: Production, Crystal Structures, Properties, Applications, 4th edn, Wiley-VCH.

18. Martinez K, Cupitt J, Saunders D, Pillay R (2002) Ten years of art imaging research. Proc IEEE 90, 28-41.

19. Liu H, Huang M, Cui G, Luo MR, Melgosa M (2013) Color-difference evaluation for digital images using a categorical judgment method. J Opt Soc Am A 30, 616-626.

20. Liang H (2012) Advances in multispectral and hyperspectral imaging for archaeology and art conservation. Appl Phys A 106, 309-323. 